- Define, where possible, sustainable ecological targets for lake restoration

- Identify and provide early warning of new threats

- Generate cause-effect hypotheses for ecological change, especially in relation to the impact of multiple stresses

- Evaluate steady-state and dynamic model output

Whilst useful methods for reconstruction based on high resolution core sampling, relatively accurate chronologies, multi-proxy analyses and reasonably robust transfer functions already exist, LIMPACS hopes to identify where critical further methodological improvements are needed. It will also encourage a move towards a more holistic paleoecological approach within paleolimnology that is concerned with ecosystem responses and interactions as well as environmental reconstruction. Entirely new techniques for dating and analysis may also be needed and it will be essential to explore GIS and model-based approaches for upscaling to regional and global scales. It will also be necessary to work closely with the LUCIFS and HITE communities especially in exploring relationships between lake change and lake catchment change.

\section{Progress so far}

LIMPACS is still in its formative stages. A steering group* of paleolimnologists, limnologists and modellers has been set up and a science implementation plan is being developed. The next step is to set up a series of working groups. It is envisaged that each will be concerned with processes associated with specific threats or stresses as follows:

- acidity, sulphur and nitrogen

- uvb radiation, dissolved organic carbon

- nutrient enrichment, phosphorus and nitrogen

(i) deep lakes, oxygen (ii) shallow lakes, trophic interactions

- salinity, ionic composition and hydrological change

- warming, stratification and mixing

- pollution, toxic metals and organics

- sediment infilling, catchment erosion

- introduced species

Further information is available on the LIMPACS web-page: www:// geog.ucl.ac.uk/ecrc/limpacs. Anyone interested in taking part in LIMPACS should contact Rick Battarbee or Cathy Stickley (c.stickley@ucl.ac.uk).

\section{Rick Battarbee}

Environmental Change Research Centre, University College London, UK

r.battarbee@ucl.ac.uk

* Steering group members are: John Anderson, John Smol, Brian Rippey, Peter Dillon, Colin Reynolds, Erik Jeppesen, Rick Battarbee, Dan Engstrom, Mike Binford, Peter Gell and Marten Scheffer.

\title{
Ecosystem Processes and Human Dimensions - The Scope and Future Of HITE (Human Impacts on Terrestrial Ecosystems)
}

\section{Introduction and Rationale}

Ecologists are increasingly aware that a complete understanding of ecosystems may require the observation and analysis of ecosystem functioning over relatively long time-scales. This may be seen from three perspectives. First, information about the causes, rates of change and direction of long term ecological trajectories may show how modern terrestrial ecosystems are conditioned by past interactions between human activities and the natural environment. Second, analysis of past ecosystem processes and rates of change may provide at least partial analogues for present and projected responses to human impact and climate change. Third, long ecological time-series may allow calibration and evaluation of predictive ecological models.

For these purposes, information about past ecosystems gained through direct observations, monitoring and measurement will often be either of insufficient length or not available. This limits the value of such an approach both for documenting and understanding the processes involved in terrestrial ecosystem change, as well as for developing and validating relevant models. The over-riding goal of HITE is therefore to further the use of environmental archives for documenting and understanding terrestrial ecosystem change through time; thereby improving the scientific basis for ensuring the security and enhancing the value of terrestrial ecosystems for the future. The scope must embrace not only human impacts but also climate variability, since the two interact. It must also be firmly rooted in our understanding of ecological principles and processes derived from studies of contemporary systems.

The initiative seeks to define and to promote research on key issues relating to human impact and natural environmental change. Paleo-ecological research has a long tradition, but paleo-ecological findings have often been under-exploited in terms of their input to our understanding of terrestrial ecosystems. Within the context of
PAGES, this reflects the extent to which research so far has laid stress on climate systems. In this type of formulation, paleo-ecological evidence is used almost exclusively as a basis for inferring climate by means of transfer functions of various kinds. This fails to accommodate paleo-ecology in its own right, that is to say, reconstructing the nature of past ecosystems through evidence independent of that used to infer climate change. Only by doing this will it be possible to understand, without recourse to circular argument, the complex of environmental factors and processes responsible for ecosystem development, modification, or demise. An additional impediment to a fuller development of paleo-ecology as we understand it has been the tendency for too many studies to use singly proxies (for example pollen analysis) as the sole basis for inferring past ecosystem change. Just as paleo-climate research has come to rely on a wide range of mutually constraining proxies, so must paleo-ecology learn to benefit from the 


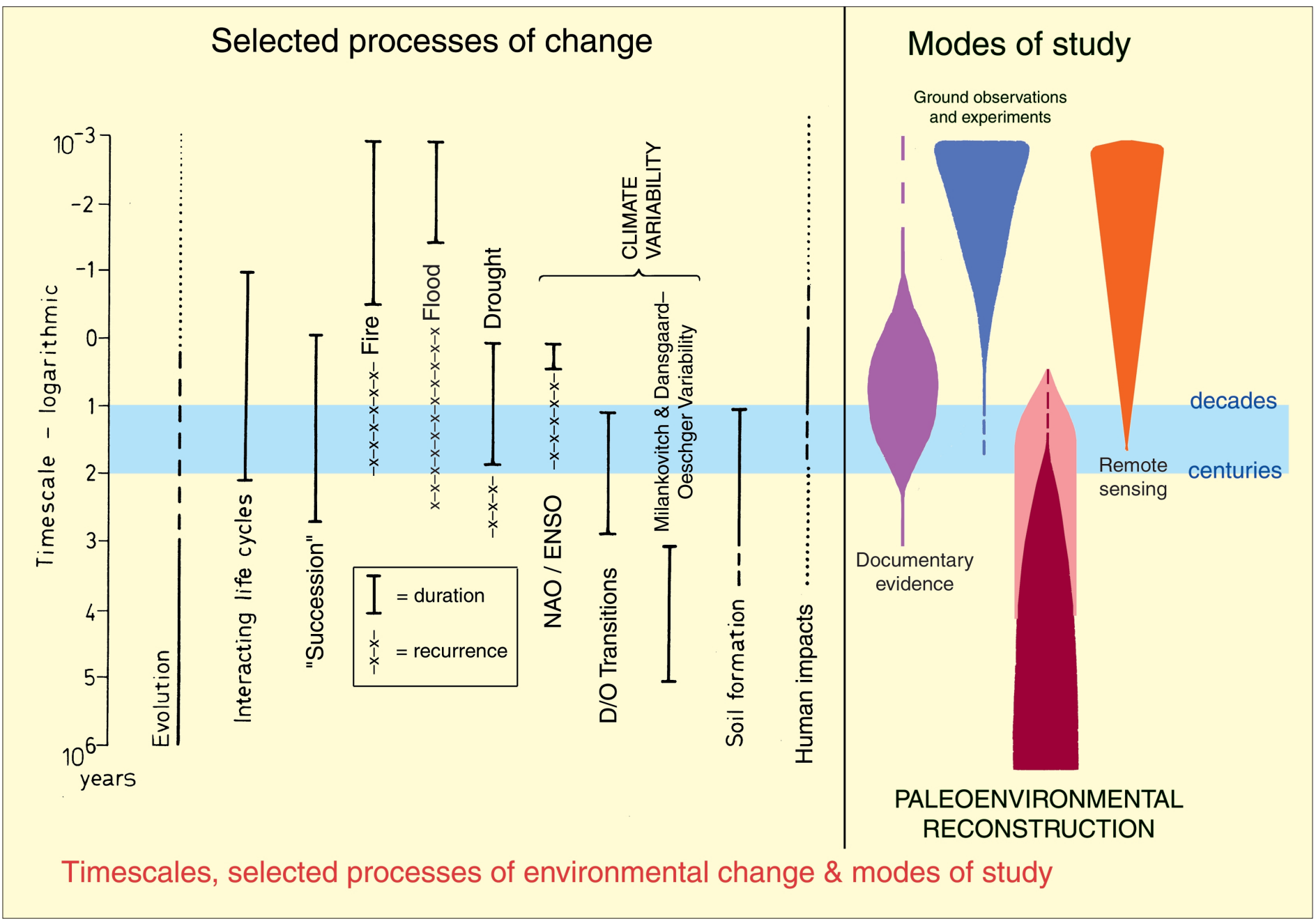

Figure 1 sets out the timespan over which a range of environmentally significant processes, both natural and anthropogenic, operate, against a sketch of the time frames with which different modes of study are mainly concerned. The vertical scale is logarithmic and refers to time frames, not to time elapsed before the present day. Thus processes with a short time frame may have operated in both the recent and the remote past. For fire, flood and drought, both duration and likely recurrence interval are indicated.

growing range of techniques, many of them sediment based, that complement pollen analysis and shed light on other aspects of past ecosystem structure and function.

Up until recently, within the broader context of IGBP, there has been a discontinuity between PAGES research and that on contemporary and future terrestrial ecosystems led mainly by GCTE. HITE aims to promote rigorous and integrated methodologies in which ecologists, modelers, environmental historians and paleo-ecologists may work profitably together. Figure 1 attempts to highlight the need for linking shortand long-term research as well as to capture the potential synergy inherent in the linkage.

The questions addressed by HITE focus explicitly on the responses of terrestrial ecosystems to the combined effects of human activities and natural environmental variability mainly during the second half of the Holocene, the period when human impact begins to interact with and eventually dominate the course of ecological changes in many of the long settled areas of the world. The goal is to produce output for the wider ecological and earth systems communities, conservation bodies, and land use managers and policy-makers. At site, watershed and regional level, key questions include the following:

- What have been the major human impacts (driving forces) that have influenced the ecosystems that we see at the present day?

- How have these interacted with natural environmental variability?

- To what extent and in which ways are the changes brought about by the combination of human and natural influences threatening the future functioning of terrestrial ecosystems systems?
- What evidence does the past record provide as a guide to resilience, rates of recovery, irreversibility and future sensitivity?

- How realistic is it to retain any concept of 'natural' ecosystems?

- In the absence of such a concept, how may evidence about past conditions help to develop realistic policy and management targets?

Where major human impacts have been long-term and sustained, the concept of 'natural' ecosystems may be inadequate. Nevertheless, 'natural' processes are operating even in the most intensively managed ecosystems, and evidence about the past dynamics of processes in ecosystems are essential to increase our understanding of the dynamics of terrestrial ecosystems and, ultimately, to develop realistic policy and management targets.

In addition to the above questions that are applicable at the local to regional 
level, there are more fundamental questions about ecosystem dynamics that paleo-data may help to address, for example:

- What can we learn about the dynamics of ecosystems which include, and in many cases are dominated by long-lived taxa?

- Is it possible to use the paleo-record to develop generalizations regarding the nature of ecological thresholds and non-linear changes?

- In so far as future climate scenarios for given ecosystems are sufficiently similar to past conditions, what does the paleo-record tell us with regard to survival and persistence at species, patch and landscape levels?

- In environments where long term succession is likely to influence future ecosystems, what does the paleo-record tell of successional processes, rates and trends?

- Where the periodicity/mean recurrence interval of extreme events or disturbance regimes is decadal or longer, what insights do paleo-data give on frequencies, impacts and interactions?

- What do studies with high temporal resolution during and after ear- lier periods of rapid environmental change tell us about rates of biotic response?

- In light of all the above, how can the paleo-record best interact with ecosystem modeling?

\section{Scientific Contexts and Case-study Inte- gration}

The above questions cover a wide range of environmental contexts and research agendas and will be addressed mainly through site-specific case-studies leading where possible to generalizations about generic properties of particular sets of ecosystems

There is now an urgent need for us to select exemplary case-studies that have general significance for generating and testing hypotheses, that are likely to provide answers to important ecological questions and that represent extensive, valued or vulnerable ecosystems. In addition, there is an equally urgent need for a series of shared goals, priorities, criteria and research protocols that will provide an integrative framework for the case studies undertaken. HITE proposes to hold an initial Workshop in Spring 2001 to begin the process of articulating and developing the necessary research agenda. We envisage, at this stage, a group of around 20 colleagues representing key case studies which we propose to unite into a coherent research framework through the definition and adoption of common goals, priorities, criteria and protocols.

\section{Frank Oldfield}

PAGES IPO, Bern, Switzerland

frank.oldfield@pages.unibe.ch

\section{John A. Dearing}

Department of Geography, University of Liverpool, UK i.dearing@liverpool.ac.uk

\section{Marie-José Gaillard}

School of Biosciences and Process Technology, Växiö University, Sweden

marie-jose.gaillard-lemdah|@ibp.vxu.se

\section{Harald Bugmann}

Mountain Forest Ecology, Department of Forest Sciences, Swiss Federal Institute of Technology Zurich, Switzerland

bugmann@fowi.ethz.ch

\section{RefERENCES}

Oldfield, F. (1983) Man's impact on the environment: some recent perspectives. Geography, 68, 245 256.

Roberts, N. (1989;1998). The Holocene. (Editions 1 and 2), Blackwell, Oxford.

\section{Using History To Interpret Current Environmental Conditions and Future Trends: An Example from the US Long Term Ecological Research (LTER) Program}

Acknowledgement of the value of a historical framework for modern studies has led major US agencies including the Forest Service and National Park Service to employ a wide range of retrospective approaches to define historical ranges of variability in landscape patterns and ecosystem structure and function as a basis for conservation and natural resource planning. Similarly, the 25 sites in the US National Science Foundation's Long Term Ecological Research (LTER) Program increasingly emphasize historical research involving scientists from diverse social and physical disciplines. Examples from the Harvard Forest LTER site in Massachusetts underscore the essential contribution of history to environmental research and planning. Importantly, historical studies figure into all aspects of this research program, from interpreting modern vegetation patterns and developing ecological restoration strategies to projecting the future role of temperate forests in the global carbon balance to anticipating forest response to atmospheric nitrogen deposition.

Like all of the New England states and much of the eastern US, Massachusetts has undergone a remarkable transformation over the past 300 years ${ }^{11}$. Following European settlement in the $17^{\text {th }}-18^{\text {th }}$ centuries, land was increasingly cleared for agriculture into the mid $19^{\text {th }} \mathrm{C}$ when $70 \%$ was open pasture and arable land and the remainder was largely coppice stands and woodlots. With industrialization and the development of mid-western agriculture in the $19^{\text {th }}$ C, New England farmland was abandoned and the rural popula- tion joined a rapidly growing immigrant population in cities, industrial towns, and, more recently, the suburbs ${ }^{23}$. Neglected farmland reforested naturally, and with wood products increasingly supplied from elsewhere, tree size, forest age, and wood biomass are on an upward trajectory. New England is currently $50-90 \%$ forested $^{12}$.

Although ecosystem studies in the eastern US have generally proceeded with little consideration of historical factors, Harvard Forest research underscores the message that incorrect interpretations occur without a detailed knowledge of changing landscape conditions and environmental drivers over past centuries or millennia ${ }^{14}$. At a local scale, forest composition and structure in New England are strongly linked to prior use of sites as for example, pas- 